\title{
A CASE STUDY OF THE SENTENCE FINAL PARTICLE "BA" 'S PRAGMATIC FUNCTIONS IN CHINESE-SPEAKING CHILDREN
}

\author{
Di Zhang', Zhibo Wang ${ }^{2}$, Robert Elliott ${ }^{3}$ \\ Cognitive Science and Linguistic Research Center, School of Foreign Language, Linyi \\ University, Linyi, China \\ zhangdi79@foxmail.com¹,wangzhibo66@126.com²,rbrt_lltt@yahoo.com³
}

\begin{abstract}
The process of the language acquisition of children is reflected in two aspects; language structure and pragmatic functions. Data from "The Longtime Tracing Oral Corpus of Typical Development Children" (one child; 2,367 sentences) are analyzed. This study examines the child's sentence final particle (SFP) "ba", in which we focused on the intention of this word and concluding with the core pragmatic meaning and divided the different levels of "ba". In this way, we combined the different sentence types, the intentions of utterance, and the degrees of "undetermined intention" and researched on the pragmatic function of child's SFP "ba" in order to obtain the pragmatic acquisition of "ba" at the specific development period (1;12-4;01). The acquisition and development of children's language is the result of a combination of biological factors, discourse input, and cognitive ability. Finally, the enlightenment of the research conclusions on early childhood education are pointed out.
\end{abstract}

Keywords : SFP “ba;” intentions; pragmatic function; sentence types; language acquisition

\section{INTRODUCTION}

The pragmatic function of a language refers to the fact that languages are used in interpersonal communications. To children, the ability to communicate with people is of utmost importance (Russell \& Grizzle, 2008). The research on pragmatics (e.g., context, discourse, utterances, and etc.) differs from traditional language research (pronunciation, grammar and meanings), and studies involving children's pragmatics and conversation analysis have been conducted for more than 50 years. Ninio and Snow (1996) indicate their work in the book, Pragmatic Development, is a milestone on children's pragmatic studies, turning the research from sparse works to full and systematic studies. Current research on children's language acquisition and pragmatics have even surpassed past theories of traditional ways of acquisition (Piaget, 1923) and social culture (Vygotsky, 1986). Research has changed its focus away from behaviorism (Skinner, 1957), universal grammar (Chomsky, 1965; 1987; Brown, 1973), those that are based on rules (Bloom, 1970; MacWhinney, 1982), usages (Rowland et al., 2003; Tomasello, 2003; Ambridge \& Lieven; 2015; Rowland et al., 2003), and systematic and functional linguistics (Fine \& Freedle, 1983; Halliday, 1975), to socialized pragmatic studies (Ochs \& Schieffelin, 1979; 1983; Romaine, 1984; Schieffelin \& Ochs, 1986) and children's interactive studies. In 
general, the process of children's language acquisition is reflected in the following two aspects: language structure and pragmatic functions. The development of children's language acquisition can be regarded as their languages being developed subjectively and objectively. Moreover, the subjective development is children's ability of socialization. From this, we can see clearly the significance of the research that has been conducted on children's pragmatic function.

The development of children's pragmatic function includes mainly the development of sentence types and communicative intention. Particles can mark the functions of a sentence, and the use of particles is related to the social relationship of both parties and the attitude of the speaker. So, we may say that one of the most effective ways to research children's pragmatic functions is through the use of particles. The main focus of this paper is on the pragmatic function of a part of speech ${ }^{1}$ in Chinese - the SFP "ba", which researchers abroad seldom study. More specifically, this study drills down to children's final particles in both the Japanese (Matsui et al., 2016; Matsui \& Yamamoto, 2013; Murasugi, 2013) and Korean (Clancy, 1989; Choi, 1991) language experiments and diaries from the perspectives of grammar and pragmatics. Studies in China found mostly in masters' dissertations (Li, 2005; Liu, 2009; Qian, 2003; Tao, 2012) and papers on pragmatics of children's SFPs are quite rare (Li, 2012).

Therefore, this paper, based on children's corpus, made a thorough study of the SFP "ba," which analyzed the intention of "ba" and tried to answer the following questions: (1) What intention do the child have with the SFP "ba" at a certain age? (2) What is the acquisition order of "ba"'s intention? and (3) Is there a relationship between the acquisition order of "ba"'s intention and the core intention or sentence types?

\section{METHOD}

\section{The Participant of the Research}

The corpus of this paper comes from the longitudinal tracking of a child from the age of one year, three months to four years old, which belongs to the corpora, titled "The Longtime Tracing Oral Corpus of Typical Development Children ${ }^{2} . "$ In this study, the child was born to an ordinary family located in Northern China, with normal intelligence, and without any cognitive disorder. His parents' education, career, and family situation have no special case.

\footnotetext{
${ }^{1}$ There is a tone of Indo-European languages, which is often expressed by the prosody including intonation, stress, and so on, but there is without a part of speech to express the tone. Typical sentence-final particles are including /a/, /ba/,/ma/,/de/,/le/ ect. for examples from $\mathrm{Li} \&$ Thompson(1981):
(1) ta chu qu mai dongxi le (2) ta hen hao kan ba
he exit go buy thing SFP s/he very good look SFP

"He's gone shopping." "S/he's very good looking, don't you agree? " "How are you?"

${ }^{2}$ This corpora is a sub-item of "the Children's Multi-modal Oral Corpus" in the Cognitive Science and Linguistic Research Center of the School of Foreign Language in Linyi University which is built by the international general standards CHILDES (Child Language Data Exchange System). The first author and her team members are responsible for collecting corpora and building it. The purpose of the corpus is to serve children's language research in southeastern China. The data used in this paper is from "the Longtime Tracing Oral Corpus of Typical Development Children". And it's authorized by the corpus ownerthe School of Foreign Language of Linyi University. We use video recorders to collect a corpus weekly. Each videotape was lasting an hour without any interruption. The content usually was the free exchange of children, parents and their companions (Xie \& Zhang, 2017).
} 


\section{Transcription}

All conversations were audio-taped using high-fidelity equipment (Panasonic HD camera: HDC-HS900GK) and transferred by two research assistants using the ELAN (EUDICO Linguistic Analysis) and the CHAT Transcription Format (MacWhinney, 2009), and the human verification was also applied. The statistic gathering of the number of times was done according to token. The examples from repetitions and nursery rhymes were not taken into consideration. The first appearance is the time when this word was first output correctly by children. The acquisition time is the time when the word was output correctly by children for three times together.

\section{The Research Method}

We mainly use natural the observation method and the case-based longitudinal research method to describe a child's daily discourse and try to analyze these sentences from the perspective of linguistics and education. The observation method mainly refers to the regular observation of the target child through recording or video in daily life in order to observe the language development of the target child and his/her psychological state or behavior pattern.

The corpus collected in the natural state of daily communication is true and reliable. And the child development principle is also of reference value.

The case-based longitudinal study method is a method of continuous follow-up investigation of the research object. The language development of children is dynamic. Through the longitudinal survey, a comprehensive language development material can be obtained, which can more clearly observe the details of language changes in each period and really reflect the development of children's language.

\section{The Acquisition Situation of "Ba"}

In this research, the acquisition of "ba" can be seen in Table $1^{3}$. This child first produced "ba" independently in $1 ; 09 ; 26^{4}$ and acquired imperative usage of "ba" in 1;12;02. In the whole process of tracking, "ba" appeared a total of 2,367 times.

Table 1: The Acquisition Situation of " $b a$ " in Case of WMX

\begin{tabular}{|c|c|c|c|c|}
\hline Total Times & Age of First Appearance & \multicolumn{3}{|c|}{ Age of the Acquisition $(1 ; 12)$} \\
\hline \multirow[t]{4}{*}{ ba (2367) } & pa ba & wa ba & zou ba & zou, zou ba \\
\hline & crawl SFP & dig SFP & walk SFP & walk walk SFP \\
\hline & "Crawl." & “Dig!" & "Walk!" & “Let’s walk!”. \\
\hline & $(1 ; 09 ; 26)$ & $(1 ; 11 ; 11)$ & $(1 ; 12 ; 02)$ & $(1 ; 12 ; 02)$ \\
\hline
\end{tabular}

However, existing studies suggest there are two pragmatic classifications of sentences. One way is conducted according to sentence types (declarative sentences, interrogative sentences, imperative sentences and exclamatory sentences) and another is according to intentions, which

\footnotetext{
${ }^{3}$ We use the first letter of the name to refer to the child.

${ }^{4}$ We take the form of age; month; day to express the time of children's language acquisition.
} 
is common in child language acquisition (Kong \& Li, 2008). Both classifications have their own advantages and disadvantages. The first classification is clear and easy to understand and is a common method among academics, but it is easy to misunderstand them as mood and sentence types. The second classification is a certainly rational, but is very difficult to have a uniform standard among academics. Therefore, this paper will use a combination of both classifications. First, sentences with the SFP "ba" were divided into four categories: declarative sentences, interrogative sentences, imperative sentences and exclamatory sentences. And then, these sentences are analyzed within each category according to their intentions.

After checking the corpus, we found that WMX were used with "ba" in three sentence types: declarative; interrogative; and imperative sentences, and there were two other kinds of answering sentences and abbreviated question sentences ${ }^{5}$. Details can be found in Table 2 .

Table 2. The Situations of Appearance of " $b a$ " in Case of WMX

\begin{tabular}{|c|c|c|c|c|c|}
\hline No. & $\begin{array}{l}\text { The sentence types } \\
\text { of appearance }\end{array}$ & Samples & $\begin{array}{l}\text { Age of First } \\
\text { Appearance }\end{array}$ & $\begin{array}{l}\text { Age of the } \\
\text { Acquisition }\end{array}$ & $\begin{array}{l}\text { Frequencies of } \\
\text { Appearance }\end{array}$ \\
\hline \multirow{3}{*}{1} & Imperative & pa ba & $1 ; 09 ; 26$ & $1 ; 12 ; 02$ & 1037 \\
\hline & Sentences & crawl SFP & & & \\
\hline & & “Crawl." & & & \\
\hline \multirow{6}{*}{2} & Abbreviated & shi ba & $1 ; 12 ; 11$ & $2 ; 01 ; 13$ & 605 \\
\hline & Question sentences & yes SFP & & & \\
\hline & & "Is it ?" & & & \\
\hline & Interrogative & shi zhe ge ba & $1 ; 12 ; 28$ & $2 ; 01 ; 29$ & 486 \\
\hline & Sentences & yes this SFP & & & \\
\hline & & "Is this?" & & & \\
\hline \multirow{6}{*}{3} & Answering & hao ba & $2 ; 01 ; 13$ & $2 ; 02 ; 17$ & 161 \\
\hline & Sentences & ok SFP & & & \\
\hline & & “'Ok.” & & & \\
\hline & Declarative & Ta shuo jin lai ba & $2 ; 02 ; 28$ & $2 ; 03 ; 14$ & 78 \\
\hline & Sentences & he say come in SFP & & & \\
\hline & & "He said came in." & & & \\
\hline
\end{tabular}

Why does "ba" not appear in the exclamation sentence? The exclamatory sentence is used by the speaker to express feelings, and human emotions are expressed through exclamatory sentences with the exclusive experience of self-examination and self-evidence. In other words, this emotion belongs to the speaker himself and cannot be shared, discussed, or consulted. Finally, the feature of exclamatory sentences is opposed with the core intention of "ba" (undetermined), therefore, "ba" does not appear in the exclamatory sentence.

\footnotetext{
5 Answering sentences are refer to these simple sentences structure just use "ok" "right" "all right" etc following the SFP "ba" in order to show the reluctantly response of speakers. Abbreviated question sentences are refer to a kind of fixed format just like hao ba? (ok SFP?), dui ba? (right SFP?) and xing ba? (alright SFP?) in order to express euphemistically asked and looking forward to a positive answer.
} 


\section{Results Intentions of "Ba"}

\section{Imperative sentences with "ba".}

We start from the corpus and analyze WMX's imperative sentences with "ba", which has six types: order; request; suggesting; urging; helplessness; and impatience.

\section{Order.}

(1) (WMX wanted to eat a mango.)

$\mathrm{CHI}^{6}$ : gei wo nong kai gei wo nong kai ba give me do open give me do open SFP "Open it for me, open it for me."

(2) (The adults were pressing for water, WMX wanted to press and could not do it.)

CHI: mama ni ya ba

Mom you press SFP

"Mom, you press."

In the academic circle, some hold the belief that "ba" cannot express orders, the reason being that an order is given when the speaker has clear intention and this conflicts with the core meaning of "ba". We checked the corpora and found some imperative sentences expressing orders would use "ba." From the perspective of pragmatics, the stiff attitude of the imperative sentences was softened after "ba" was used, making the sentence easier to be accepted. For example, in (1), WMX wanted to eat a mango and he used "for me," which was a structure expressing strong orders. To soften the stiff order and to fit in with his low social status, "ba" is used at the end of the sentence so his mother could help him eat the mango smoothly. In (2), WMX's father was pressing for water, and WMX felt it was novel and, so he tried to press and found that he was not strong enough to get water. He gave an order to his mother and let his mother press. Here, if "ba" were not used, it would be impolite and could not achieve the ease of mitigation caused by orders.

\section{Request.}

(3) (WMX wanted to take his bear and go to another room with his mother.)

CHI: xiong xiong \# xiong xiong \# zou ba \# ma ma\# zou ba bear \# bear \# go SFP \# Mom \# go SFP

"Bear, bear, Let's go. Mom, Let's go."

(4) (WMX wanted to go out and play so he send out asking.)

CHI: en en \# zou ba \# chu qu wan wan ba ok ok\# go SFP\# go out play play SFP "Ok, Ok, Let's go out!"

Because the child is younger, and his social status is not high in the conversation, his requests

\footnotetext{
${ }^{6} \mathrm{CHI}$ is the symbol of the transfer system on behalf of the child, MOT on behalf of the mother and \# on behalf of a short pause.
} 
contained some sort of negotiation and begging, in which "ba" was used. Here, "ba" expressed the intention that he wanted to be noticed and indicated some degree of earnest. If "ba" were not used, then the meaning was strong and earnest was not expressed. When expressing request and negotiation, "ba" shows the speaker's subjective intention. For example, in (3), WMX wanted to go to another room with his mother, and so he sent a request to his mother. To emphasize the goal to go to another room with his mother, he used "ba" to soften the compelling tone to finally realize his purpose. In (4), to increase the degree of politeness, WMX repeated the word "play" and added "ba" to reduce the trouble that his action might bring to his mother.

\section{Suggestion.}

(5) (WMX suggested that his mother lie down on the bed and wanted to play in bed.)

CHI: ni tang xia ba \# ma ma you lie down SFP

"You lie down, Mom."

(6) (WMX suggested while riding a bike.)

CHI: ma ma zan lia qi ba mom we two ride "Mom, let's ride!"

Children use the suggestion function very often when expressing an action they wanted to accomplish, because this function reduces the possibility of being rejected and in this way, their purpose may be successfully realized. For example, in (5) and (6), purposes of WMX were to play in bed and ride a bike with his mother, and he therefore suggested his mother lie down and ride a bike. In this way, he accomplished his communication intentions indirectly. Suggestions and request have similar pragmatic function, and the focus is to express the subjective intention of the speaker. The difference lies in the fact that request comes from the perspective of the self and the suggestion, the perspective of others, but their purposes are the same.

\section{Urging.}

(7) (Papa's phone was ringing, and WMX urged.)

CHI: kuai jie dian hua ba quickly answer the phone SFP

"Quickly answer the phone!"

(8) (WMX urged his mother to play with him.)

CHI: ma ma \# ni kuai gen wo \# wo wan ba mom \# you quickly with me play \# me play SFP "Mom \# quickly play with me \# with me."

Children's urging function appears when they are the leading position in the communication. The urging function itself indicates "impoliteness" and so children use "ba" to soften this indication and make up for the lack of politeness. For example, both in (9) and (10), WMX used "quickly" to express a hurried sense and in the end of the sentences, he used "ba" to soften 
the tone and thus dilute the "impoliteness". He did this to reduce the conflict in communication.

\section{Impatience.}

(9) (WMX wanted to drink something quickly.)

CHI: gan kuai ba

quickly SFP

"Quickly."

(10) (The recorder had been going on for a long time. And the temperature of the recorder was high.)

CHI: na \# na \# na \# ni hai shi bie xian lu le ba then \# then \# then you still not first record SFP

"Then \# then \# then you do not record it."

\section{Helplessness.}

(11) (WMX wanted to stop recording but his mother insisted.)

CHI: wo hai shi zi ji wan ba

I still myself SFP

"I'd better play by myself."

(12) (WMX could not find his slippers.)

CHI: na wo hai shi zhe yang guang zhe jiao ba

then I still such bare feet SFP

"Then I'd better bare my feet."

The last two functions appeared late, which can be attributed to the fact that the children's individual characteristics started to appear with the growth of children. And they have the ability to express the dissatisfaction with their own language. However, limited by the principle of "politeness" in communication, they tend to use "ba" to dilute the conflict when they express a kind of negative emotion such as impatience and helplessness. For example, in (10) (11) and (12), WMX used " 'd better" with a kind of modality meaning "non-resolute," which echoed the compromise and hesitation of "ba".

The six types of intentions in the above imperative sentences can be further divided into three types of pragmatic functions - function of suggestion (suggestion, request), function of order, function of urging (urging, impatience, and helplessness). The core intention of "ba" is "undetermined ${ }^{7}$," which shows most clearly in the function of suggestion. But, children are still in the process of language development and there are not many adverbs appearing together. And thus, the pragmatic effects of expressing euphemism and improving the degree of politeness are carried out by "ba". The function of suggestion mainly expresses the subjective desire of the speaker. The speaker, out of his self-interests, asks others to do things for him which could easily bring about troubles to others. So, to increase the degree of politeness, in the case of less coexisting adverbs, children may apply repetitions of verbs to express the

\footnotetext{
7 The core intention of the word "ba" is "undetermined" according to "On the distributional verification and semantic extraction of the sentence final particle ba" (Zhao \& Sun, 2015).
} 
meaning of "trying", which echoes with "ba" for example, "go out play play SFP". Therefore, children use the euphemistic method to reach their purpose in the communication between a junior and a senior.

In the order function, which expresses authority or forcefulness, "ba" is seldom used. Conversely, "ba" often functions as easing up the tone in children's imperative sentences when expressing orders. It expresses an attitude of non-forcefulness and relief of the loss of politeness in the orders. In the urging function, the degree of "undetermined" is very low and the attitude of the speaker is definite and "ba" only alleviates the degree of rigidness. As mentioned above, the core pragmatic function of "ba" in imperative sentences is to weaken the tone, and its core intention of "undetermined" varies in degrees with the language context, the purpose and the function of communication, and the degree of the speaker's subjectivity. In the functions of suggestion and order, the core intention "undetermined" are expressed in certain degrees; in the function of urging, the core intention is diluted, and its main function is to adjust the tone and the emotion of the speaker.

\section{Interrogative sentences with "ba".}

"Ba" in children's language mainly appears in abbreviated question sentences and interrogative sentences. Following is our detail analysis of intentions with "ba" of WMX. There are four major types.

\section{Guessing.}

(13) (Grandma and WMX looked at pictures. WMX pointed to a picture and guessed.)

CHI: shi jie jie ba \# ai \# shi jie jie ba is sister SFP \# ai \# is sister SFP

"Is she a sister? \# ai \#Is she a sister?"

(14) (Mother was telling a story, which concerned some things, and WMX guessed the name of one of them.)

CHI: mian bao ba

bread SFP

"Is it a bread?"

Children use "ba" to show his own speculation on the proposition information to verify or consult from the other person. Here, the core intention "undetermined" of "ba" is getting highlighted. In fact, in the mind of the child, there is already a default answer, but he is still waiting for the other person's answer. For example, in (13) and (14), WMX already gave guesses on "sister" and "bread", and he still expected answers from his grandma and his mother. He gave the right of answering to the other person with asking and seeking opinions.

\section{Doubt.}

(15) (WMX pissed pants, and asked to have pants replaced.)

CHI: zen me shi shi de \# bu hui shi niao le ba why is wet \# not will be piss SFP

"Why is it wet \# Did I piss pants?" 
(16) (WMX wanted to have bubble gum. He wanted to blow out bubbles, but he could not do it.)

$$
\begin{gathered}
\text { CHI: mei you ba } \\
\text { no have SPF } \\
\text { "No bubbles?" }
\end{gathered}
$$

It is quite rare for children to use "ba" to express doubt. They usually have judgments in their mind, but to avoid being too arbitrary and considering both sides of the "politeness" problem, they use "ba" to realize the politeness function. For example, in (15), WMX might have already known that he pissed pants, but to have a way out, he used doubt tone and denial form to express the fact that he pissed pants. First, he put the surface phenomenon out "Why is it wet?" to be followed by the key point afterwards. In (16), WMX wanted to blow bubbles from the gum, but the fact differed from his expectation, and therefore, he used "ba" to express his intention of doubt.

\section{Seeking Agreement.}

(17) (WMX wanted to express that his mother was not angry and seek the same feeling from his mother.)

CHI: ma ma ni zhe hui er bu sheng qi dui ba mom you now not angry right SFP

"Mom, you are not angry, are you?"

(18) (WMX wanted to play with building blocks and his mother said several holes were not plugged.)

$$
\begin{gathered}
\text { CHI: jiu zhe yang ba } \\
\text { then such SFP } \\
\text { "That's it?" }
\end{gathered}
$$

When children asked for agree, they often use declarative sentences with some fixed language forms such as "right SFP?" "that's it SFP?" "all right SFP?" "true SFP?" to strengthen the effect of politeness, and they do so hoping to get the other person's agreement so that they could do things according to their wish. In (17), WMX did not want his mother to become angry, he used negative format and fixed form "right SFP?" to express his opinion, hoping that his mother agreed with his presupposition "not angry" and thus realized the purpose of language communication. In (18), WMX wanted to stop playing blocks and he used "ba" to ask for agreement, hoping his mother could give up building blocks and accompanied him to play other toys.

\section{Begging and Requesting.}

Zhou (2009) believed, "It is common in languages of the world to beg and request with questions, and now it is more common to express euphemism or suggestion with an undetermined question." For example,

May I have your name, please?

In children's language, such form is also used to express some additional intention "negotiation, suggestion, urging and asking" etc. 
(19) (WMX did not want to look at pictures, and negotiated with his mother to let her tell a story.)

CHI: gei wo jiang gu shi ba give me tell story SFP

"Could you tell me a story?"

(20) (WMX suggested going to Mickey Park.)

CHI: qu qu mi qi gong yuan qu ba go go mi qi park go SFP

"Shall we go to Mickey Park?"

(21) (WMX wanted to watch TV and asked his mother's opinion.)

CHI: zan men kan dian shi ba ma ma we watch TV SFP

"Shall we watch TV, Mother?"

(22) (WMX massaged his father and then asked his father a question.)

CHI: shu fu ba comfortable SFP

"Are you comfortable?"

(23) (WMX urged to finish recording.)

CHI: jie shu le ba

finish SFP

"Finished?"

The four types of intention of the interrogative sentences with "ba" can be further classified into three kinds of pragmatic functions - euphemistic function (guessing and doubt), seeking agreement function and begging and requesting function. In the language context, interrogative sentences with "ba" do not have very high degrees of questioning and sometimes they are only tones of guessing. Guesses and doubts can also be classified into euphemistic function. However, they are still kinds of "interrogative sentences" and therefore the degree of "undetermined" are higher than those of imperative and declarative sentences. These expressions with "ba" do not affect the truth condition of such sentences, but make the speakers' judgment not so definite and the tone more eased-up so that the meaning of the speaker is less strong. Interrogative and declarative sentences with "ba" can both express seeking agreement function. The speaker already has an expected reply to his question, but to avoid losing the listener's face or for the sake of politeness so he uses "ba" which superficially looks like asking for the listener's opinions but in fact asking for agreement. Children in communication often are in inferior positions and so they often use seeking agreement function of interrogative sentences with "ba" (reflected in fixed forms). Begging and requesting function appears often in imperative sentences with "ba" and to use the form of interrogative sentences to express the imperative intention, that is to say, apply the imperative sentence with "ba" and plus the interrogative tone. This usage often comes with the suggestion function of imperative sentences. The "undetermined" degree of such imperative sentences are higher than that of interrogative sentences. All in all, the core intention of euphemistic function and begging and requesting function of interrogative sentences is "undetermined" or "uncertain." Seeking agreement function on the surface is also expressed "uncertain", but the ultimate goal is to let the other 
person to agree with own view of the speaker and not really expressed uncertainty about his own words.

\section{Declarative sentences with "ba".}

The frequency of usage of the declarative sentence with "ba" is the lowest, and it can express guessing, helplessness, answering and asking for common ground. Following is our detailed analysis of the language intention of the declarative sentences with "ba" of WMX, and they can be grouped into four types.

\section{Guessing.}

(24) (WMX asked where his scooter was and then he said.)

CHI: ke neng zai da xue li ba maybe at university SFP "Maybe it's at the university."

(25) (Sound came from outside, and WMX guessed it was singing.)

CHI: ta men shi chang ge de sheng yin ba they are sing sound SFP "They are the sound of singing."

Both declarative and the interrogative sentences may express guessing but the meaning of guessing of the declarative sentence is not as strong as that of the interrogative sentence and the guessing of the declarative sentence is close to euphemistic expression and asking for common ground. For example, in (24) the adverb "maybe" and "ba" were to express the guessing of WMX himself and then he gave a confirm repetition. Though the "ba" expressed guessing, this guessing expressed the fact that he already had an answer, and such sentence has a lower degree of "undetermined."

\section{Helplessness.}

(26) (WMX was counting but he was not willing to do so, and he expressed helplessness.)

CHI: wo zi ji shu ba I myself count SFP "I will count by myself."

(27) (WMX wanted his mother to open the oven for him, but his mother did not do it and, so he was helpless.)

CHI: da kai lu zi \# wo zi ji da kai ba open the oven \# I myself open SFP "Open the oven \# I will open it by myself."

Compared with the intention of helplessness, we can find that when children express helplessness with "ba," they often use "I" as a subject and the feeling they expresses is not related to that of the listener. This discourse phenomenon is often monologue. In fact, children in the early stages of language acquisition often use a lot of monologue. But, SFPs usually have stronger emotions and this is quite obvious in pragmatics and therefore what we selected for material is more of communication. In the specific language context of helplessness, all 
the children expression is a kind of helplessness about their limited ability. This becomes "unwilling compromise", a monologue to express a kind of particular emotion about self.

\section{Answering.}

(28) (WMX wanted to open the window and looked out.)

MOT: There is sunlight, right?

CHI: hao ba

yes SFP

"Yes."

(29) (Discussing what toys to take when watching the movie.)

MOT: You keep it \# keep it \# till next week when I bring you to see a movie and then take it.
CHI: na hao ba
that ok SFP
"That's Ok."

When expressing the intention of answering, children often use fixed forms such as "Ok SFP" "That's Ok SFP" and "That's all right SFP" to express their certain kind of approval. This approval is not a direct one but a reserved one. But, in our material, we only find the expressing "Ok SFP" and nothing else. Liu (2009) finds that there is only one sample "That's all right SFP" of answering and appears quite late $(3 ; 04 ; 28)$ in JBS's language. The reason is that modal adverbs and SPF appear at the same time, which happens only in a later period of children language development.

\section{Seeking Agreement.}

(30) (At night, WMX was playing with a toy car. His mother said the noise would make the uncle downstairs unhappy. He changed the method and wanted to continue to play in another way. He then gave the right to his mother to decide. From the surface, he was asking for the opinion of his mother but in fact, he was asking his mother to agree with him and let him continue to play with the toy car.)

CHI: zhe yang ye hai xing le ba this also still ok SFP

"It is Ok in this way."

(31) (WMX was playing with blocks. He wanted other colors, so he expected his mother agree with him and he did not want more purple ones.)

CHI: tai duo zi se le ba

too many purples SFP

"There are too many purple blocks."

Interrogative and declarative sentences with "ba" both have intentions of seeking agreement. On the surface, the speaker is asking for the listener's opinion but in fact, the speaker in the case of his own default answer is asking for approval and to reach an agreement finally. For example, in (30) and (31) the presuppositions were "keep playing" and "want blocks of other colors" and WMX was eager to reach his goal. If WMX directly asked each other to do 
according to his own wish, it would be impolite and not conform to the child's lower social position. Therefore, the form of asking for opinions was appeared in order to ask for approval, in other words, seeking agreement.

Helplessness and answering are both of the euphemistic function of "ba", and the core meaning is the "undetermined." With "ba" at the end of the sentences, original meanings of sentences are not changed. That is to say "ba" did not change the "affirmation" into "doubt." In fact, sentences with the effect of the "euphemism" has a low degree of "undetermined." Children use "ba" in answers to show that they are not willing to carry out each other's request right away, expressing "unwillingness of compromise", but at the end, children agree with each other. The function of seeking agreement, seen from the aspect of interpersonal relations, is asking for opinions from the surface, but in fact, is asking the other party to agree with them. So, the degree of "undetermined" is very low.

In the above, we analyze the pragmatic type of SFP "ba." The time of their first appearance and acquisition are shown in Table 3.

Table 3. The List of intentions of SFP "ba" in Case of WMX

\begin{tabular}{|c|c|c|c|c|c|c|}
\hline \multirow{2}{*}{$\begin{array}{l}\text { Sentence Types } \\
\text { Imperative }\end{array}$} & \multicolumn{6}{|c|}{ Intentions (First Appearance time / Acquisition Time) } \\
\hline & Suggestion & Request & Order & Urging $2 ; 05 ; 02 /$ & Impatience & Helplessness \\
\hline \multirow[t]{2}{*}{ Sentences } & $1 ; 12 ; 11 /$ & $2 ; 01 ; 29 /$ & $1 ; 09 ; 26 /$ & $2 ; 05 ; 23$ & $3 ; 07 ; 12 /$ & $3 ; 12 ; 21 /$ \\
\hline & $1 ; 12 ; 18$ & $2 ; 02 ; 03$ & $1 ; 12 ; 11$ & & $3 ; 12 ; 28$ & $3 ; 12 ; 28$ \\
\hline Interrogative & Guessing & Doubt & Seeking & Begging & Abbreviated & \\
\hline \multirow[t]{3}{*}{ Sentences } & & & Agreement & Requesting & Questions & \\
\hline & $1 ; 12 ; 28 /$ & $2 ; 05 ; 15 /$ & $2 ; 09 ; 29 /$ & $2 ; 02 ; 03 /$ & $1 ; 12 ; 11 /$ & \\
\hline & $2 ; 01 ; 29$ & $2 ; 07 ; 24$ & $2 ; 09 ; 29$ & $2 ; 03 ; 22$ & $2 ; 01 ; 13$ & \\
\hline Declarative & Guessing & Helplessness & Answering & Seeking & & \\
\hline \multirow[t]{3}{*}{ Sentences } & & & & Agreement & & \\
\hline & $2 ; 11 ; 25 /$ & $2 ; 12 ; 17 /$ & $2 ; 01 ; 13 /$ & $3 ; 09 ; 29 /$ & & \\
\hline & $2 ; 12 ; 21$ & $2 ; 12 ; 21$ & $2 ; 02 ; 17$ & $3 ; 12 ; 13$ & & \\
\hline
\end{tabular}

According to the previous analysis, we divide the degree of "undetermined" of the core intention of the SFP "ba" into three levels - high, medium and low, and further analyze sentence types and intentions according to three levels. About this analysis, please refer to Table 4. The highest level of "undetermined" lies in "interrogative sentence" and "abbreviated question sentences", by which children only take the form of question and do not really ask something. They are "questions with affirmative answers", but in all of the core intention, they have the highest level of "undetermined." The lowest level of "undetermined" lies in seven types of intentions such as "impatience, urging and helplessness" in declarative sentences and imperative sentences. The four intentions of declarative sentences are all "affirmative answers with doubts", but the prerequisite is "affirmative answers" which can also be regarded as a strong psychological pre-planning. The children with "impatience, urging, and helplessness" in imperative sentences also have strong psychological pre-planning, and the initiative of the communication is in the children and they only use "ba" to buffer the discomfort of a strong 
psychological pre-planning. In this way, the degree of "undetermined" falls to the lowest position. The "order, suggestion and request" in the imperative sentences are in the medium level. When children express such language intentions, there is some uncertainty in their mind, and their psychological pre-planning is not very strong, with a kind of negotiation. Children usually use these medium level sentences with SFP "ba" in the conversation. The appearance of SFP "ba" comes in the time order of imperative sentences $>^{8}$ interrogative sentences + abbreviated question sentences $>$ declarative sentences + answering sentences. From the "undetermined" degree of SFP "ba", the medium comes first, then the highest, and finally, the lowest.

Table 4. The Core Pragmatic Level of SFP "ba" in Children

\begin{tabular}{|c|c|c|c|}
\hline \multirow{5}{*}{$\begin{array}{l}\text { The Degree of } \\
\text { "undetermined" }\end{array}$} & High & Medium & Low \\
\hline & Interrogative Sentences & & Declarative Sentences \\
\hline & & Imperative Sentences : & Imperative Sentences : \\
\hline & Abbreviated Questions & Order, Suggestion, Request & Impatience, Urging, \\
\hline & & & Helplessness \\
\hline
\end{tabular}

The "request" in imperative sentences and the "guessing" in interrogative sentences and abbreviated question sentences come almost at the same time. The "order and suggestion" of imperative sentences; declarative sentences (aside from answering) and the "impatience, urging and helplessness" of imperative sentences are at the opposite ends (early and late) of the degrees of "undetermined." Why do children not first gain interrogative sentences with higher level of "undetermined"? The reason is the complexity of sentence types: imperative sentences are easier to obtain than interrogative sentences. The "request" in the imperative sentences and the "guessing" in interrogative sentences and abbreviated question sentences appear almost at the same time and this explains the fact that because of the high degree of "undetermined," children quickly obtain the "guessing" intention in interrogative sentences and abbreviated question sentences with SFP "ba" after they acquire the sentence type of interrogative. The declarative sentences and the "impatience, urging and helplessness" of imperative sentences are acquired at a later time and this is decided by the pragmatic property of the low "undetermined" level. And then, both being imperative sentences, why are they obtained in two different levels? That might be attributed to the fact that with the children growing, they can accurately express their dissatisfaction emotion. Also, with the growing level of socialization, controlled by the principle of politeness, when expressing negative moods, there is strong subjectiveness in children's language with the low degree of "undetermined", they will still choose "ba" to weaken potential conflicts.

\section{DISCUSSION}

Focusing on this idea presented at the beginning of the paper: (1) Intentions of SFP "ba" are mainly distributed in imperative sentences (6 types of intentions), interrogative sentences (5 types of intentions), and narrative sentences (4 types of intentions) according to sentence types,

\footnotetext{
8 We borrow the symbol "> " to indicate "earlier than".
} 
children under four years old have already basically grasped the major intentions of SFP "ba," which shows that they have gained basic social communication skills after gaining basically elements of language at three years old. Although these pragmatic skills are still simple, direct, and subjective, children are able to think in another person's perspective ${ }^{9}$ and use a "polite" communication strategy. (2) The order of acquiring intentions of SFP "ba" is: imperative sentences $>$ interrogative sentences + abbreviated question sentences $>$ declarative sentences + answering sentences and a more detailed order of acquisition is not repeated here. (3) The order of intentions of SFP "ba" is related to the core intention "undetermined" and sentence types. This order is the result of the combined effect of the core intention and sentence types. The syntax, semantics and pragmatics of children's language are the process of comprehensive acquisition and development. It is not feasible to conduct the isolated study separately. While it is true that children must obtain certain language ability before they can communicate adequately, many studies show that children have already acquired the ability to interact and communicate in the pre-language period. Therefore, only when we combine the elements of sentence types, context, the intention of language and pragmatic strategy can we get the full blueprint of children's language.

We take sentences with SFP "ba" as the key of our study and make a detailed and comprehensive analysis on intentions of "ba" . The children that we study started to acquire the basic usage of SFP "ba" at 1;12 and the language intention still developed till 4;01, although the language intention at this period displayed various and socialized interactive features. The language structure of children's expressions is becoming more complex (with the appearance of particles), and this trend is decided by the clarity demand of communication; at the same time, children's language expressions are becoming finer (with the appearance of multiple language intentions) and this trend is decided by the degree of socialization of language communication. On the surface, intentions of "ba" have a tendency of diversification, in fact this intention is closely related to the core "undetermined" which combines with sentence types in affecting children's acquisition of the pragmatic function of SFP "ba" . The pragmatic function of "ba" indicates the germination of children's socializing consciousness and they start to realize their own weakness of communication - younger, lower social status and less discourse power. So, in language communication, they purposefully take a euphemistic way and give more power of decision to the other party to avoid conflicts. In this way they can realize their intentions. Together with their maturity, children's pragmatic ability will grow until they become real social individual.

According to our research, children approaching four years old $(3 ; 08)$ will use the SFP "ba"'s intention to seek common ground, which is to consider others' perspectives. In this situation, declarative sentences are often added with fixed language forms such as "right SFP?" "that's it SFP?" "all right SFP?" "true SFP?", to strengthen the degree of politeness. This can be considered as a primitive era of "the second-order theory of mind", which conforms to relevant

\footnotetext{
${ }^{9}$ Psychologists think typical development children already have the Theory of Mind (ToM) at the age of 4 . ToM is the ability to predict and understand one's own and others based on the state of desire and belief. Judging from the literal meaning of the speaker only need the first-order theory of mind, while judging the potential intentions of the speaker need the second-order theory of mind. In the stage of the first-order ToM, one only needs to know other's thoughts. In the stage of the second-order ToM, one must know other's thoughts from the other person's point of view.
} 
research result which believes that four-year old children have already obtained "the secondorder theory of mind" (Bowler \& Strom, 1998; Angeleri \& Airenti, 2014). As far as typical development children are concerned, the three-year olds have already grasped the basic skills to use particles, and yet, their acquisition of pragmatic functions has just started and will continue until they reach adolescence. But four-year old children have already acquired part of the pragmatic skills, for example, the application of "the principle of politeness" and "the function of euphemism." Yet, children of this period still cannot grasp the higher-level pragmatism. For example, their understanding of figurative language, such as "irony" and "metaphor." Children at three or four years old have all entered kindergarten, and so their teachers should pay attention to the skills of using particles. For example, they can use "ba" to inspire the children. They can say, "You stand up and answer this question SFP?" and "This is a drawing of a rabbit SFP?" The teachers also need to know they should not use too many particles for purposes of figurative language expressions, especially not using "irony" to praise or criticize children. A bad example is when the teacher said to a child "Look how busy you are SFP! (ironic compliment)" when a child actively helps the teacher putting toys aside.

For developmental disorders children such as autism spectrum disorder, mild learning disabilities, attention-deficit /hyperactivity disorder, and intellectual disabilities, their period of language acquisition comes later than typical development children. So, the application of research results on children with these developmental disorders children should be postponed considerably to the next or later development period. Exactly how long the period is needs to be categorized according to specific situations and yet, it is concluded that the research results gathered from typically developed children are not suitable for children with developmental disorders. This is to say that children three to four years old with developmental disorders children do not have the pragmatic skill of particles or their skill is too basic. Therefore, teachers and parents of special education children should inspire and attract children specifically at three and four years old on the basic usage of particles. When doing this, the use of higher level pragmatic skills should be avoided. The process should go on until developmental disorders children reach "the second-order theory of mind," when the particle's social function (the pragmatic skill) is combined into daily language communication and language teaching. At this time, the intervention can also be done on specific language skills. For example, irony is always a problem for developmental disorders children; they cannot obtain the potential intention which is opposite to the surface meaning. In this situation, we can combine our research results with the understanding of irony, to make the SFP as a language clue to irony. We can design some cases of the use of irony so that we can guide and intervene such children's pragmatic acquisition, to let them gain new social skills or reject improper behaviors. In this way, they might reduce the probability of being laughed at or bullied and will enhance their ability to adapt to society.

Because the collection of the corpus is not yet complete (lasts to 2018), the age group of the case is limited to the child under four years old. Future studies should increase the number of children's cases and the corpus extended to six or seven years old when children's pragmatic development is fuller. On this basis, the further analysis of intentions of the SFP "ba" should be accomplished and the results of the case study should also be extended to more children for 
comparative analysis to make the findings more valid and generalizable.

\section{Funding}

This research is a periodic result of a Shandong Social Science Planning and Research Project (No. 16CZWJ27) and is sponsored by "the United Board for Christian Higher Education in Asia".

\section{REFERENCES}

Ambridge, B., \& Lieven., E. (2015). A constructivist account of child language acquisition. In MacWhinney. B \& W. O. Grady (Eds.), The Handbook of Language Emergence. West Sussex: John Wiley \& Sons, Inc.

Angeleri, R., \& Airenti, G. (2014). The development of joke and irony understanding: A study with 3- to 6-yearold children. Canadian Journal of Experimental Psychology, 68(2), 133-146.

Bloom, L. (1970). Language development: Form and function in emerging grammars. Cambridge, MA: MIT Press.

Bowler D. M., \& Strom, E. (1998). Elicitation of first-order theory of mind in children with autism. Autism,2(1),33-44.

Brown, P., \& Levinson, S. (1978). Universals in language usage: Politeness phenomena. In Goody, E. N. (ed.) Questions and Politeness: Strategies in Social Interaction. Cambridge: CUP.

Brown, R. (1973). A first language: The early stages. Cambridge, MA: Harvard University Press.

Choi, S. (1991). Early acquisition of epistemic meanings in Korean: a study of sentence-ending suffixes in the spontaneous speech of three children. First Language (11), 93-119.

Chomsky, N. (1965). Aspects of the theory of syntax. Cambridge: MIT Press.

Chomsky, N. (1987). On the nature, use, and acquisition of language, in W. Lycan (ed.), 1990, Mind and Cognition, Oxford: Blackwell.

Clancy, P. M. (1989). Form and function in the acquisition of Korean wh-questions. Journal of Child Language, 16(2), 323-347.

Fine, J., \& Freedle, R. (1983). Developmental issues in discourse. Norwood, NJ: Ablex.

Halliday, M. A. K. (1975). Learning how to mean: Explorations in the development of child language. London: Edward Arnold.

Kong, L. D., \& Li, H. M. (2008). The development of pragmatic functions of "le" sentence in the child language. Journal of Chinese Linguistics (13). Beijing: The Commercial Press.

Li, C., \& Thompson, S., (1981). A Functional Reference Grammar of Mandarin Chinese. Berkeley: University of California Press.

Li, H. M. (2005). Modal particles in children's language. (Master's thesis). Retrieved from http://d.g.wanfangdata.com.cn/Thesis_D513344.aspx.

Li, H. M. (2012). The development of pragmatic functions of "de" sentence in the child language and its comparison with "le" sentence. Language Teaching and Linguistic Studies (13), 105-112.

Liu, Y.J. (2009). The research of acquisition of children's modal particles “ma” and " $b a$ ”. (Master's thesis.)

Retrieved from http://www.cnki.net/KCMS/detail/detail.aspx?FileName=2009130452.nh\&DbName=CMFD2009.

MacWhinney, B. (1982). Basic processes in syntactic acquisition. In S. A. KuczajII (Ed.), Language development, 1: Syntax and semantics. Hillsdale, NJ: Erlbaum Associates. 
MacWhinney, B. (2009). Enriching CHILDES for morph syntactic analysis. Retrieved from http://repository.cmu.edu/psychology.

Matsui, T., Yamamoto, T., Miura, Y., \& McCagg, P. (2016). Young children's early sensitivity to linguistic indications of speaker certainty in their selective word learning. Lingua (175-176), 83-96.

Matsui, T., \& Yamamoto, T. (2013). Developing sensitivity to the sources of information: Early use of the Japanese quotative particles tte and to in mother-child conversation. Journal of Pragmatics (59), 5-25.

Murasugi, K. (2013). Steps in the emergence of full syntactic structure in child grammar. Nanzan Linguistics (9), 85-118.

Ninio, A., \& Snow, C. E. (1996). Pragmatic development. Boulder, CO: West view Press.

Ochs, E., \& Schieffelin, B. (1979). Developmental pragmatics. New York: Academic Press.

Ochs, E., \& Schieffelin, B. (1983). Acquiring conversational competence. London: Routledge and Kegan Paul.

Piaget, J. (1923). The language and thought of the child. In Marjorie \& R. Gabain (Eds.), London and New York: Routledge Classics.

Qian, Y.J. (2003). Modal particles in children's language. (Master's thesis). Retrieved from http://d.g.wanfangdata.com.cn/Thesis_D513344.aspx.

Romaine, S. (1984). The language of children and adolescents: The acquisition of communicative competence. Oxford: Blackwell.

Rowland, C. F., Pine, J. M., Lieven, E. V., \& Theakston, A. L. (2003). Determinants of acquisition order in whquestions re-evaluating the role of caregiver speech. Journal of Child Language, 30(3), 609-663.

Russell, R. L., \& Grizzle, K. L. (2008). Evidence based, pragmatic language competencies toward assessments. Clinical Child and Family Psychology Review (11), 59-73.

Sacks, H. (1995). Lectures on conversation (Vol. I). Oxford: Blackwell.

Schieffelin, B., \& Ochs, E. (1986). Language socialization across cultures. Cambridge: Cambridge University Press.

Skinner, B. F. (1957). Verbal behavior. New York: Appleton-Century-Crofts.

Tomasello, M. (2003). Constructing a language: A usage-based theory of language acquisition. Cambridge: Harvard University Press.

Tao, Y. (2012). The acquisition of Mandarin sentence final particles. (Master's thesis). Retrieved from http://www.cnki.net/KCMS/detail/detail.aspx?FileName=1013017099.

Vygotsky, L. (1986). Thought and language. Cambridge, MA: The MIT Press.

Xie, N. \& Zhang, D. (2017). A study on the construction of the children's multi-modal oral corpus. Technology Enhanced Foreign Language Education (177), 56-64.

Zhao, C. L. \& Sun, L. (2015). On the distributional verification and semantic extraction of the sentence final particle "ba." Studies of the Chinese Language (365), 121-132.

Zhou, S. H. (2009). Grammatical meaning and discourse function of "ba" revisited. Language Teaching and Linguistic Studies (2), 16-22. 